\title{
O SÍTIO CONTROLADO DE GEOFÍSICA RASA DO IAG/USP: INSTALAÇÃO E RESULTADOS GPR 2D-3D
}

\author{
Jorge Luís Porsani ${ }^{1}$, Welitom Rodrigues Borges ${ }^{2}$, Selma Isabel Rodrigues ${ }^{2}$ e Francisco Yukio Hiodo ${ }^{1}$ \\ Recebido em 14 julho, 2005 / Aceito em 3 janeiro, 2006 \\ Received on July 14, 2005 / Accepted on January 3, 2006
}

\begin{abstract}
The data interpretation from shallow geophysical survey can be complex because the uncertainty about subsurface targets. To reduce the ambiguity and help in the interpretation of geophysical data, the Shallow Geophysical Test Site of IAG-USP (Sitio Controlado de Geofísica Rasa-SCGR do IAG-USP) was installed inside the campus at USP. Different targets at various depths were installed along seven lines. The targets buried in the Test Site simulate targets found in geotechnical, archaeological, and environmental studies. In this article, the construction methodology of the Test Site and some GPR results along a line characterized by empty metallic tanks are presented. The geophysical characterization in controlled field condition allowed the calibration of geophysical responses due to known targets, such as, metallic tanks. $250 \mathrm{MHz}$ GPR profile showed an excellent agreement with a GPR 2D model determined by finite difference in time domain method. Fk-migration improved the resolution and the spatial position of the metallic tanks. GPR 3D results, such as 3D cube, fence diagrams, and depth slices, were very important to improve the characterization of the targets in subsurface. SCGR-IAG revealed itself as important tool for teaching, research and extension activities in university related to Geophysics, and it will be very important to our community. With the installation of this Test Site, an important step was done in searching the State of Art in Shallow Geophysics in Brazil, were the development of news equipment, modeling and inversion software could be tested and its response calibrated.
\end{abstract}

Keywords: SCGR-IAG, Shallow Geophysical Test Site-IAG, GPR-Ground Penetrating Radar, Modeling GPR 2D, Metallic tanks, Cube 3D, Fence diagrams, Depth slices.

RESUMO. A interpretação de dados de investigações geofísicas rasas pode ser complexa devido às incertezas a respeito dos alvos em subsuperfície. Para reduzir as ambigüidades e auxiliar a interpretação dos dados geofísicos de superfície foi instalado no campus da USP o Sítio Controlado de Geofísica Rasa do IAG-USP. Diferentes alvos foram instalados em várias profundidades ao longo de 7 linhas. Os alvos instalados no Sítio Controlado simulam alvos encontrados em estudos geotécnicos, ambientais e arqueológicos. Neste artigo são apresentadas as principais etapas envolvidas na construção do Sítio Controlado de Geofísica Rasa do IAG/USP e alguns resultados GPR ao longo de uma linha caracterizada por tambores metálicos vazios. A caracterização geofísica, sob condições controladas de campo, permitiu uma calibração das respostas geofísicas para os tambores metálicos instalados no SCGR-IAG. 0 perfil GPR de 250 MHz apresentou uma excelente concordância com a modelagem GPR 2D através do método das diferenças finitas no domínio do tempo. A migração-fk melhorou a resolução e o posicionamento espacial dos tambores metálicos. Os resultados GPR 3D na forma de cubo 3D, diagrama de cercas e depth slices, mostraram-se muito importantes para melhorar a caracterização dos alvos em subsuperfície. 0 SCGR-IAG constitui-se numa ferramenta importante para o Ensino, Pesquisa e Extensão Universitária em Geofísica, e será de grande importância para a nossa comunidade. Com a instalação deste Sítio Controlado, foi dado um importante passo em busca do "Estado da Arte em Geofísica Rasa" no Brasil, onde 0 desenvolvimento de novos equipamentos, software de modelagem e de inversão poderão ser testados e suas respostas calibradas.

Palavras-chave: SCGR-IAG/USP, Sítio Controlado de Geofísica Rasa-IAG/USP, GPR-Ground Penetrating Radar, Modelagem GPR 2D, Tambores metálicos, Cubo 3D, Diagrama de cercas, Depth slices.

\footnotetext{
${ }^{1}$ Departamento de Geofísica, Instituto de Astronomia, Geofísica e Ciências Atmosféricas, Rua do Matão, 1226. Cidade Universitária, 05508-090 São Paulo, SP, Brasil. Fone: (11) 3091-4734; Fax: (11) 3091-5034 - E-mails: porsani@iag.usp.br e francisc@iag.usp.br.

2 Programa de Pós-Graduação em Geofísica, Instituto de Astronomia, Geofísica e Ciências Atmosféricas, Rua do Matão, 1226. Cidade Universitária, 05508-090 São Paulo, SP, Brasil. Fone: (11) 3091-2791 ou 3091-2792; Fax: (11) 3091-5034 - E-mails: welitom@iag.usp.br e selma@iag.usp.br.
} 


\section{INTRODUCÇ̃̃O E OBJETIVOS}

0 campus da Universidade de São Paulo (USP) vem sendo utilizado como laboratório de pesquisas geofísicas desde 1993, mas somente a partir de 1997 a área em frente ao Instituto de Astronomia, Geofísica e Ciências Atmosféricas (IAG/USP) passou a ser utilizada sistematicamente como laboratório de aulas práticas.

Apesar dos estudos sistemáticos envolvendo a integração de vários métodos de investigação geofísica rasa, a interpretação dos dados pode ser complexa devido às incertezas. Para reduzir as ambigüidades encontradas na interpretação geofísica, em meados de outubro de 2003 começaram-se as obras de instalação do Sítio Controlado de Geofísica Rasa - SCGR do IAG/USP, sendo concluídas no final de novembro do mesmo ano.

O SCGR está situado na borda da Bacia Sedimentar de São Paulo, dentro do campus da USP. Geologicamente, a área é composta por um aterro, caracterizado por argila arenosa a siltosa, com alguns níveis intercalados de silte, ora argiloso, ora arenoso, predominantemente de cor vermelha escura, com espessura inferior a $3 \mathrm{~m}$. Uma cobertura neocenozóica é observada até $6 \mathrm{~m}$ de profundidade, sendo evidenciada por argilas enriquecidas em matéria orgânica. A partir daí predominam sedimentos areno-argilosos (formações Resende e São Paulo), sobrepostos ao embasamento granito-gnáissico. Dados de três poços para pesquisas geológicas-geofísicas, perfurados na área de estudos, mostram que a espessura dos sedimentos não ultrapassa $53 \mathrm{~m}$ (Porsani et al., 2004a).

A importância deste sítio controlado é que as assinaturas geofísicas de alvos cujas propriedades físicas são conhecidas serão utilizadas como respostas padrões típicas para cada tipo de material e poderão ser extrapoladas para áreas onde não se têm informações da subsuperfície. Esta situação em particular é muito interessante, pois os alvos enterrados simulam algumas situações reais em obras de engenharia e geotecnia, planejamento urbano, estudos de contaminação do meio ambiente e em pesquisas arqueológicas. Além disso, as pesquisas em desenvolvimento contribuirão para aprimorar a interpretação de dados de investigação geofísica rasa.

A instalação do SCGR-IAG é pioneira no Brasil e se insere num contexto geológico-urbano diferente de outros sítios controlados existentes no exterior. Este sítio constitui-se numa ferramenta importante para 0 Ensino, Pesquisa e Extensão em Geofísica, e será de grande importância para a nossa comunidade, consistindo num novo laboratório. Com a instalação do SCGR foi dado um importante passo para melhorar o conhecimento relativo às respostas geofísicas de alvos rasos como os encontrados nos estudos ambientais, de engenharia e arqueologia.

0 presente trabalho sintetiza a metodologia de construção do Sítio Controlado de Geofísica Rasa - SCGR do IAG/USP onde são apresentadas as principais atividades desenvolvidas antes, durante e após a instalação dos alvos no subsolo. Para ilustrar a importância da geofísica de investigação rasa na caracterização dos alvos instalados no SCGR, são mostrados alguns resultados obtidos com o método GPR - Ground Penetrating Radar sobre a linha caracterizada por tambores metálicos vazios. Dentre os resultados são apresentados os modelos GPR 2D determinados pelo método das diferenças finitas no domínio do tempo, perfis 2D (migrado e não-migrado), cubo 3D, diagrama de cercas e depth slices.

\section{ATIVIDADES DESENVOLVIDAS "ANTES" DA INSTALA- ÇÃO DO SCGR-IAG}

\section{Levantamento topográfico da área}

A área do Sítio Controlado de Geofísica Rasa do IAG é de $50 \mathrm{~m} \times$ $30 \mathrm{~m}$ e localiza-se em frente ao prédio do Instituto de Astronomia, Geofísica e Ciências Atmosféricas (IAG), no terreno do Instituto de Física da USP (Figura 1).

0 levantamento topográfico na área do SCGR-IAG, realizado antes da instalação dos alvos, teve como objetivo o mapeamento das interferências superficiais, tais como, as árvores que ocorrem dentro e nos arredores do SCGR. A localização precisa das árvores na área do sítio foi importante para o planejamento da disposiçãa das linhas contendo os diferentes alvos. Os dados topográficos foram adquiridos com uma estação total.

\section{Aquisição dos dados geofísicos}

Estudos geofísicos preliminares foram realizados nas proximidades do SCGR através da integração de dados geofísicos de superfície e de poço, e permitiram caracterizar a estrutura geoelétrica dos sedimentos e do embasamento da Bacia de São Paulo (Porsani et al., 2004a). Na mesma área foi realizado um levantamento de sísmica de reflexão rasa delimitando o topo do embasamento da bacia entre o mínimo de 35 e o máximo de $55 \mathrm{~m}$ de profundidade (Diogo et al., 2004).

Na área do SCGR-IAG, muitos perfis geofísicos foram realizados visando recuperar o background do terreno, ou seja, sem a interferência dos alvos em subsuperfície. Os métodos geofísicos utilizados foram: GPR, Elétrico e Eletromagnético Indutivo (EM38), Magnetometria e Sísmica rasa (Refração e Reflexão). Com exceção dos perfis sísmicos, os outros perfis geofísicos foram adquiridos ao longo de linhas de $30 \mathrm{~m}$, de direção N-S 


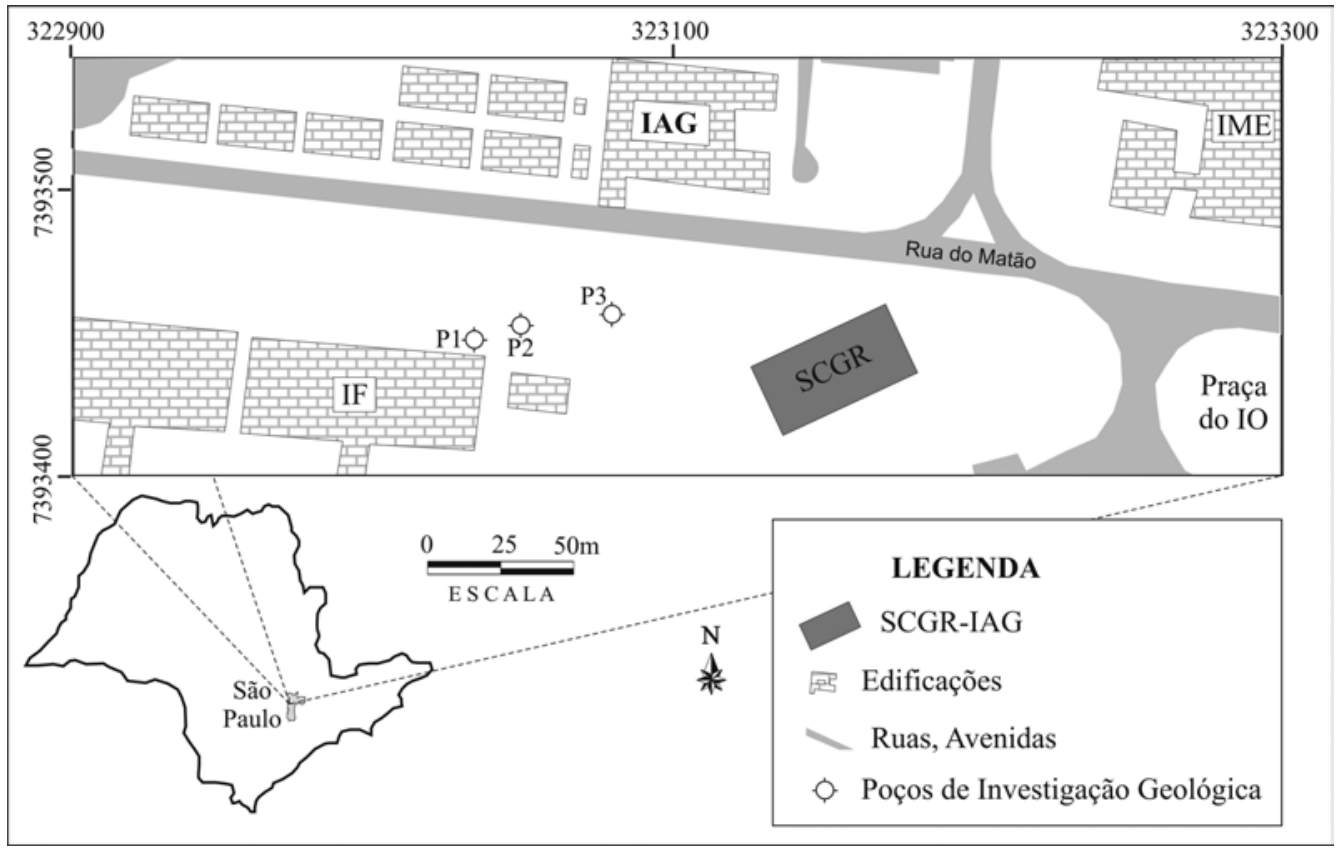

Figura 1 - Mapa de localização do Sítio Controlado de Geofísica Rasa do IAG/USP.

magnético, com espaçamento de $1 \mathrm{~m}$.

Neste artigo, são apresentados alguns resultados obtidos com o GPR. 0 GPR é um método geofisico não destrutivo, de alta resolução, que permite obter uma imagem da subsuperfície rasa da Terra, utilizando reflexões de ondas eletromagneticas de altas freqüências (normalmente entre $10 \mathrm{MHz}-2,5 \mathrm{GHz}$ ). Uma antena transmissora emite um pulso eletromagnetico para dentro da Terra. Havendo contrastes em pelo menos uma das propriedades físicas (condutividade elétrica, constante dielétrica e permeabilidade magnética) nos materiais em subsuperfície, parte do sinal é refletido, sendo recebido por uma antena receptora. 0 resultado obtido é uma imagem de alta resolução da subsuperfície, onde no eixo horizontal está a distância e no eixo vertical o tempo duplo da onda EM (ida e volta do sinal). Informações sobre este método podem ser encontradas na literatura (Davis \& Annan, 1989; Daniels, 1996; Porsani, 1999), dentre outras.

Até 0 presente momento foram adquiridos 425 perfis de reflexão GPR na área do SCGR, utilizando-se as técnicas: i) modo contínuo com antenas blindadas (100, 250 e $500 \mathrm{MHz}$ ) e não blindadas (100 e $200 \mathrm{MHz}$ ); e ii) modo passo-a-passo com antenas não blindadas (100 e $200 \mathrm{MHz}$ ). Para a aquisição dos dados no modo contínuo com as antenas não blindadas foi desenvolvido no Laboratório de Instrumentação Geofísica do IAG um trenó para transportar as antenas do GPR (Figura 2). Os dados foram obtidos utilizando-se um equipamento sueco (Ramac/Mala).

\section{INSTALAÇÃO DO SCGR-IAG}

\section{Instalação dos alvos}

A construção do SCGR-IAG seguiu os mesmos moldes do Sítio Controlado implementado na WMU - Western Michigan University (Sauck, 1996). Os alvos foram agrupados por tipos de materiais e distribuídos ao longo de sete linhas, com profundidades variando de 0,5 até $2 \mathrm{~m}$ em relação ao topo dos alvos.

Para a instalação dos alvos, o solo foi escavado utilizandose uma retro-escavadeira (Figura 3) e os trabalhos de refinamento da base dos buracos foram feitos com enxadas. Após a escavação do solo, foi realizado um levantamento topográfico para delimitar o contorno do topo e da base dos buracos escavados, visando um detalhamento da área perturbada (Figura 4). Os alvos foram instalados com orientações N-S ou E-W, e quando necessário, foram horizontalizados com níveis. Durante a disposição dos alvos, ainda com os buracos abertos, foi realizado outro levantamento topográfico visando determinar com precisão o posicionamento e a profundidade dos alvos em relação à superfície do terreno (Figura 5). Após este levantamento topográfico sistemático, os alvos foram definitivamente enterrados. Este procedimento foi repetido para cada alvo instalado no SCGR-IAG.

O SCGR-IAG é composto por sete linhas de $30 \mathrm{~m}$ cada, dispostas ao longo da direção N-S magnético. Um cano metálico de $4 \mathrm{~cm}$ de diâmetro externo foi instalado na posição de $15 \mathrm{~m}$ ao 


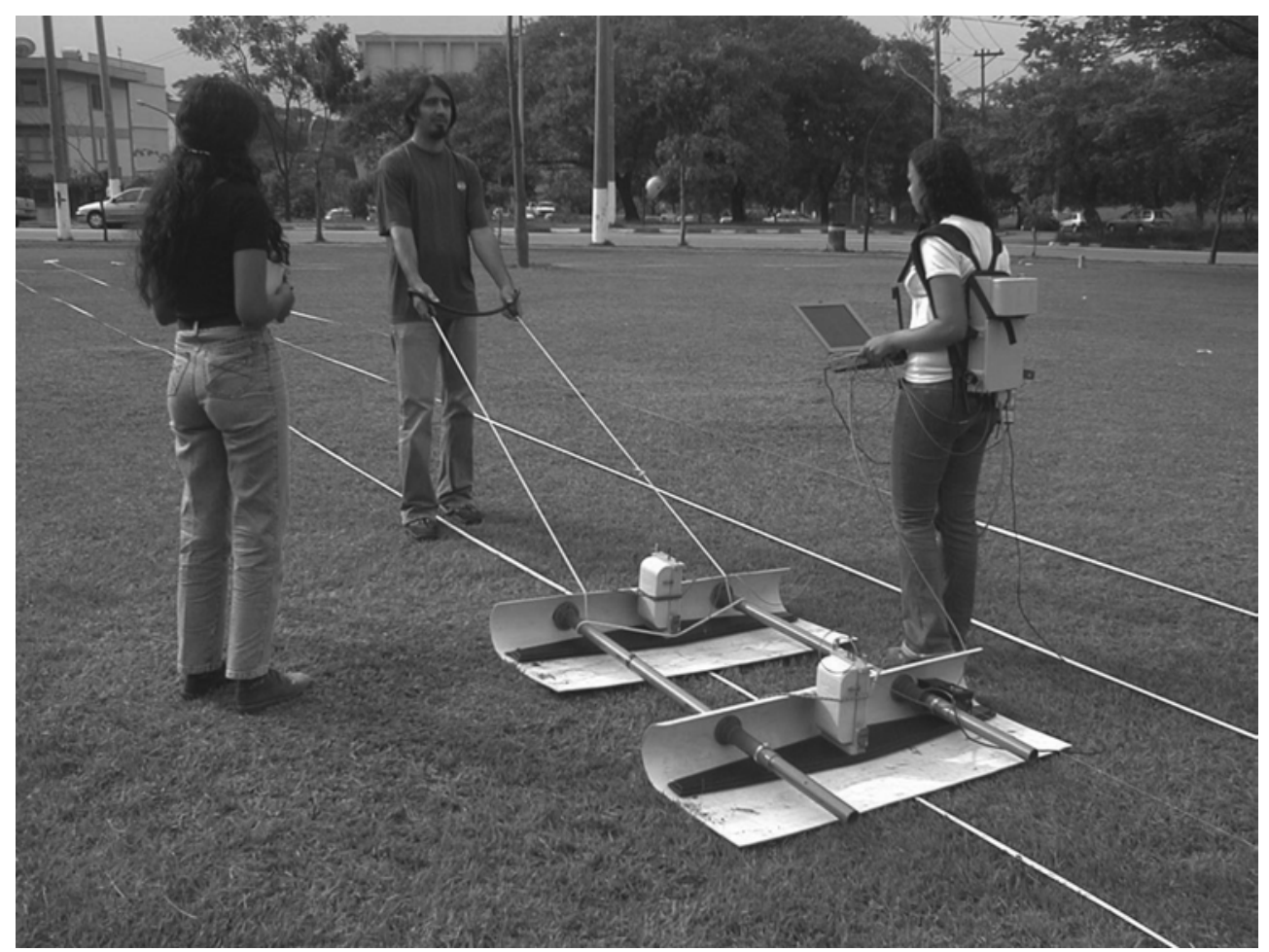

Figura 2 - Rebocador plástico para transportar as antenas GPR não blindadas.

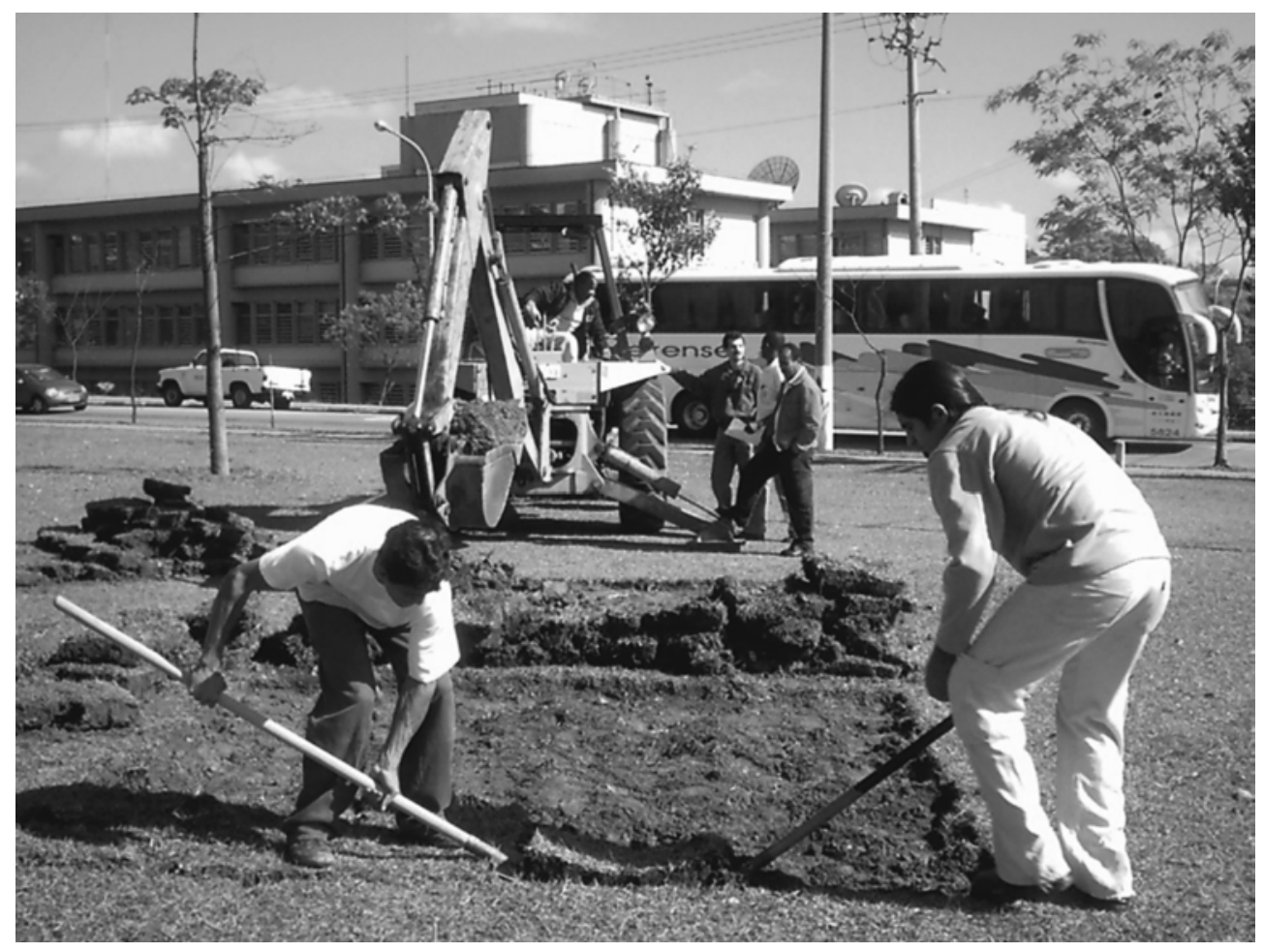

Figura 3 - Instalação dos alvos no SCGR-IAG. 


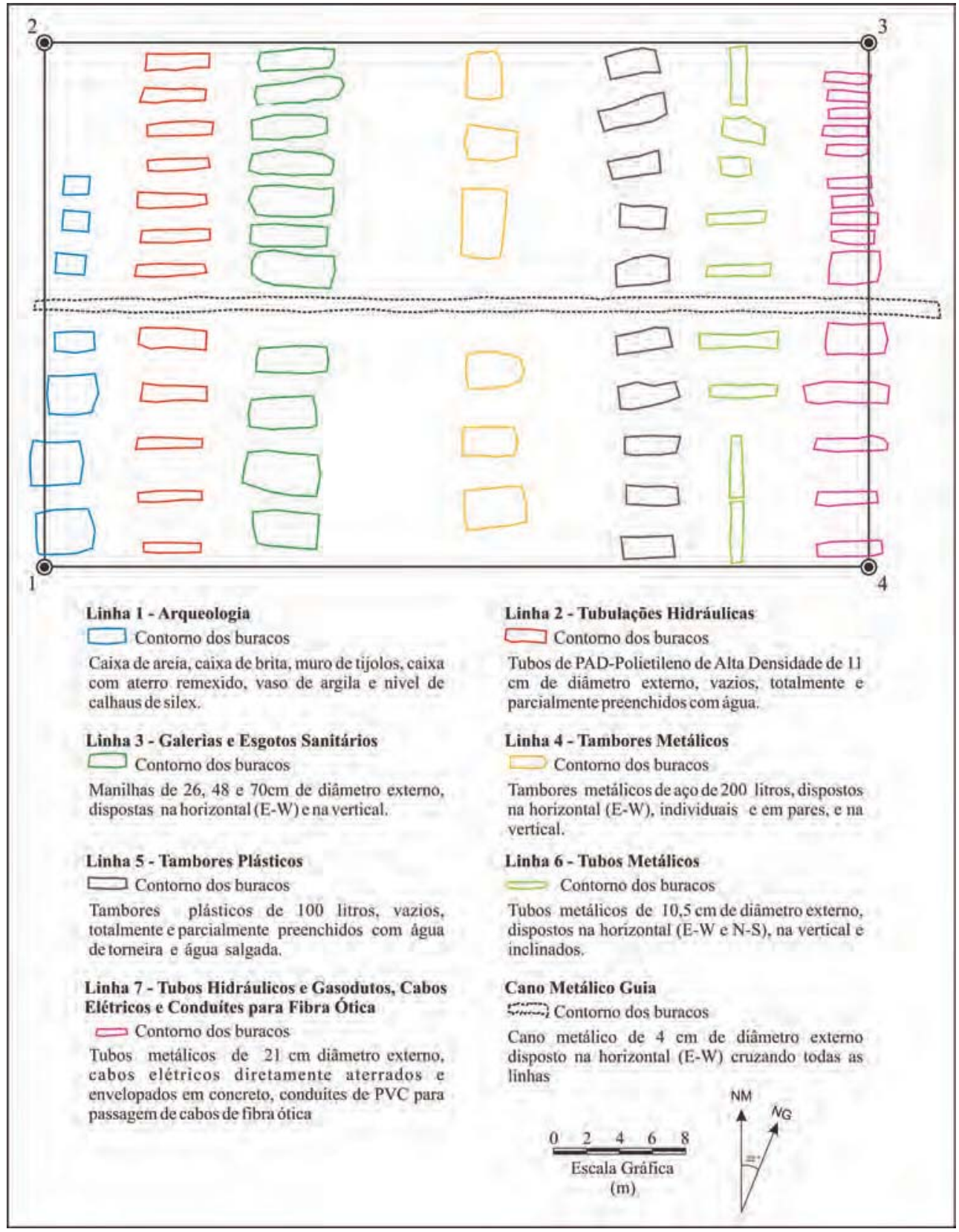

Figura 4 - Mapa de contorno da área dos buracos escavados ao longo das sete linhas instaladas no SCGR-IAG.

longo da direção E-W, servindo como alvo-guia que cruza todas as linhas instaladas no SCGR. Cada uma das sete linhas é caracterizada com um tipo específico de material. Os alvos foram escolhidos para terem respostas magnéticas, condutivas ou para gerarem reflexões nos perfis de GPR. A Figura 6 mostra o mapa base do SCGR com as marcas de controle, localização das sete linhas, o cano metálico-guia e a posição das árvores na superfície do terreno.
A Linha 1 teve como objetivo contemplar estudos arqueológicos. Nesta linha foram enterrados, em diferentes profundidades, caixas de areia e brita, solo revirado, muro de tijolos, vasos cerâmicos e um nível de calhaus de sílex. Os vasos cerâmicos simulam urnas funerárias, o muro de tijolos simula a fundação de igrejas históricas e o nível de calhaus simula uma paleofogueira.

Na Linha 2 foram instalados em diferentes profundidades tubos de PAD (Polietileno de Alta Densidade) marrom com $11 \mathrm{~cm}$ 


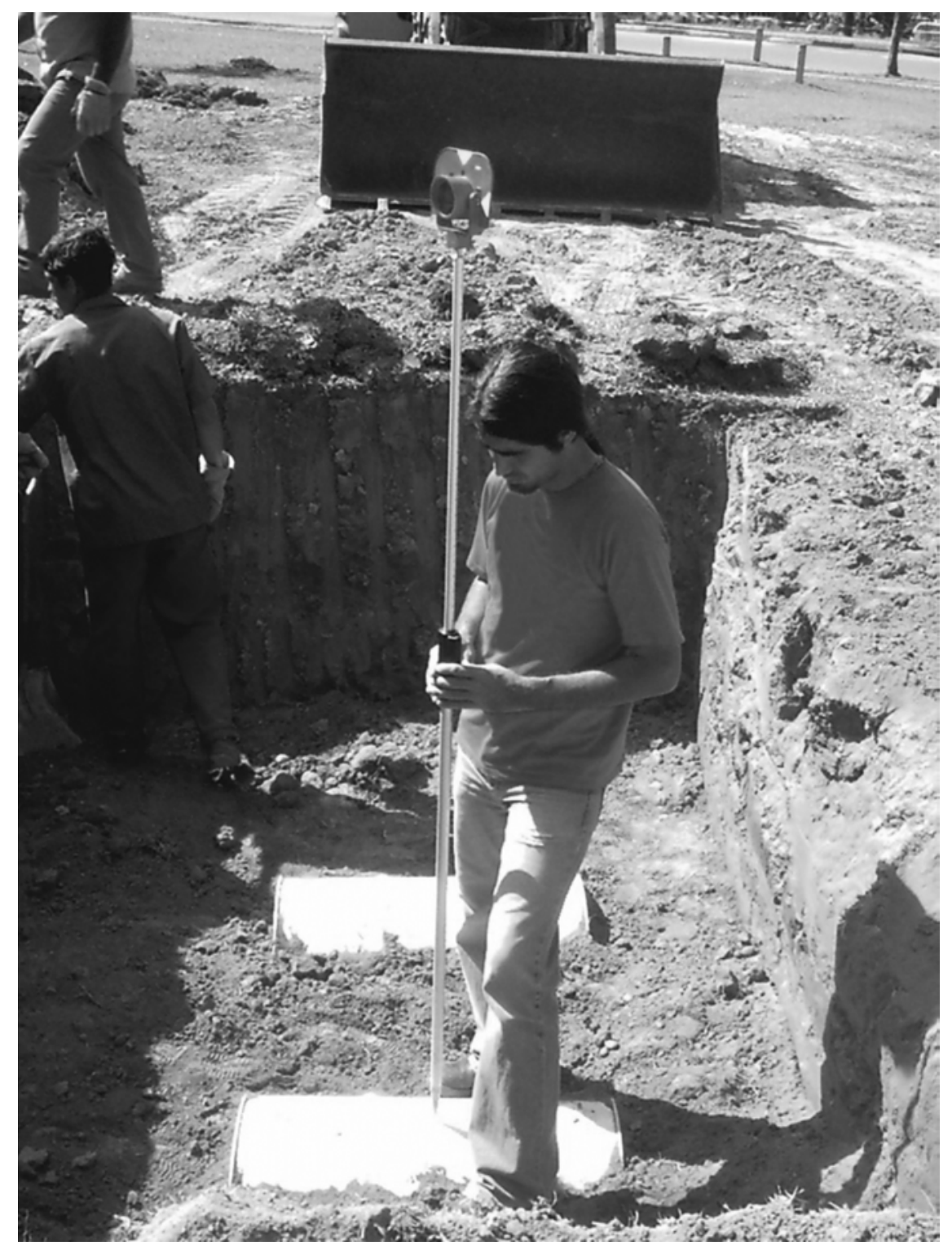

Figura 5 - Levantamento topográfico dos alvos instalados no SCGR-IAG.

de diâmetro externo e $2 \mathrm{~m}$ de comprimento. Os tubos de PAD simulam 0 transporte de água potável para as residências, sendo instalados com freqüência na grande São Paulo pela Companhia de Saneamento Básico do Estado de São Paulo (SABESP).

A Linha 3 é caracterizada por manilhas de concreto com 26, 48 e $70 \mathrm{~cm}$ de diâmetro externo, dispostas em diferentes profundidades. As manilhas simulam sistemas de transporte de águas pluviais (galerias) e o escoamento de esgoto.

$\mathrm{Na}$ Linha 4 foram instalados em diferentes profundidades tambores de aço de 200 litros, tanto na horizontal quanto na vertical. Os tambores foram pintados com tinta anti-ferrugem, visando a maior durabilidade. Todos os tambores foram enterrados vazios, para evitar problemas de corrosão. Esta linha visa contemplar os estudos ambientais voltados para a localização de tambores metálicos utilizados para descarte de produtos químicos no subsolo.

Na Linha 5 foram instalados tambores de plásticos de 100 litros, em diferentes profundidades. Os tambores plásticos também simulam problemas de contaminação do meio ambiente: contaminante resistivo (tambor vazio e tambor preenchido com água de torneira) e contaminante condutivo (tambor preenchido com água salgada e tambor parcialmente preenchido com água salgada). 


\section{Distância (m)}

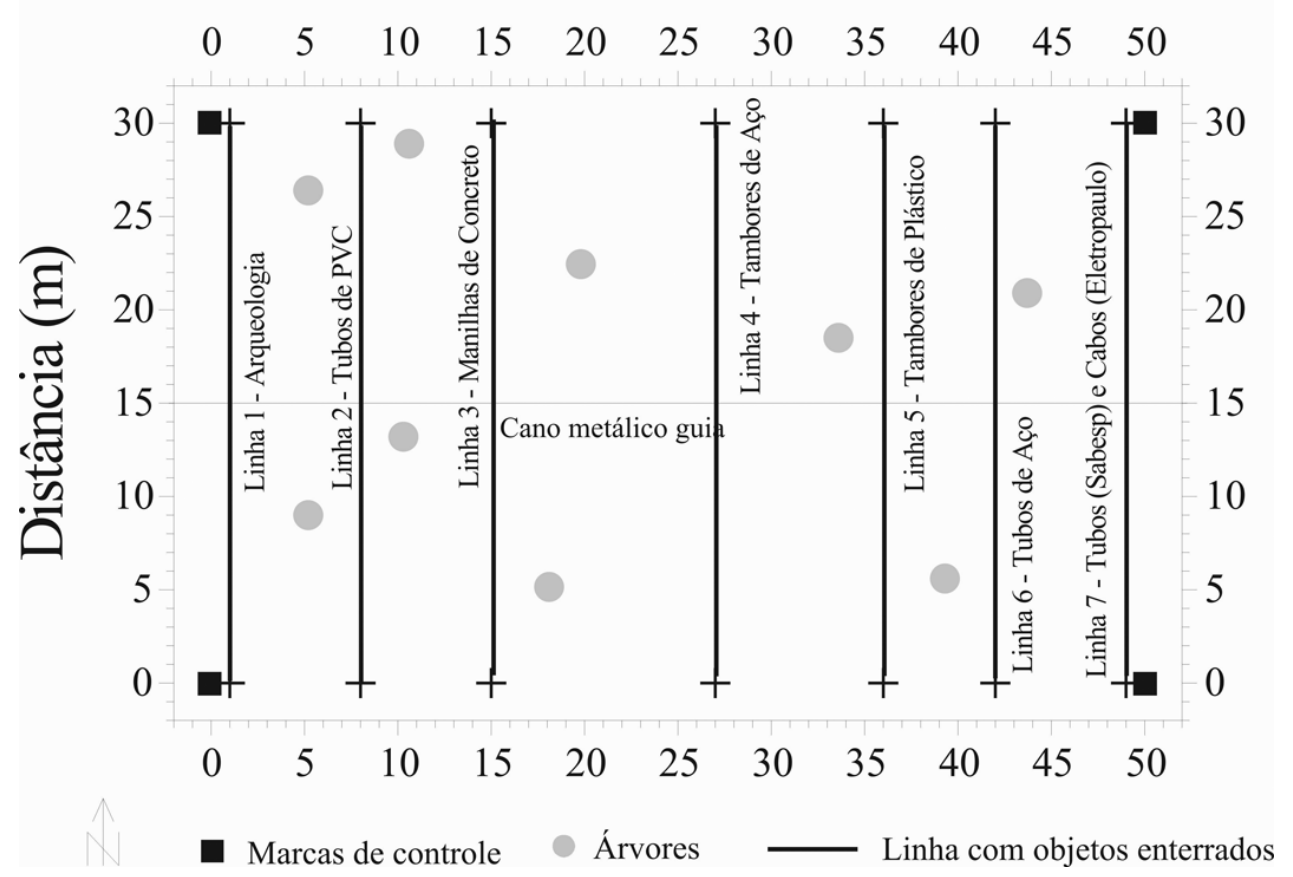

Figura 6 - Mapa do SCGR-IAG mostrando as marcas de controle, as sete linhas, o cano metálico-guia e a posição das árvores na superfície do terreno.

Para saturar a água de torneira com sal de cozinha ( $\mathrm{NaCl}$, no tambor com capacidade de 100 litros foram adicionados $20 \mathrm{~kg}$ de sal, resultando numa condutividade elétrica de $980 \mathrm{mS} / \mathrm{m}$, conforme medidas feitas no laboratório de análises químicas do Instituto Oceanográfico da USP.

Na Linha 6 foram instalados em diferentes profundidades tubos de aço com 10,5 cm de diâmetro externo e $2 \mathrm{~m}$ de comprimento. Atualmente, os tubos metálicos são usados para a passagem de gás pela Companhia de Gás (COMGAS), que abastece os edifícios, no entanto, na cidade de São Paulo, ainda existem muitas tubulações metálicas enterradas para o transporte de água potável usados pela SABESP.

Na Linha 7 foram instalados em diferentes profundidades tubulões de aço de $21 \mathrm{~cm}$ de diâmetro externo e $2 \mathrm{~m}$ de comprimento, conduítes de plástico para passagem de cabos de fibra ótica, cabos elétricos diretamente aterrados e dutos elétricos envelopados em caixas de concreto. Os tubulões metálicos simulam a passagem de gás e de água potável para os edifícios. Os conduítes de plástico servem para simular a passagem de cabos de fibra ótica usados pelas empresas de telecomunicações. Os cabos elétricos diretamente aterrados e os dutos elétricos envelopados em concreto são freqüentemente usados pela Companhia de Eletricidade do Estado de São Paulo (ELETROPAULO).
Para cada linha instalada no SCGR-IAG foi elaborada uma planilha contendo um número de identificação do alvo, o posicionamento das linhas e dos alvos, a profundidade do topo dos alvos e, uma descrição de cada alvo. Esta planilha será fundamental para os futuros trabalhos a serem realizados na área de testes controlados de geofísica rasa.

Todos os materiais instalados no Sítio Controlado visam reproduzir uma pequena amostra dos principais alvos que são normalmente encontrados no ambiente dos grandes centros urbanos e em alguns sítios arqueológicos. Com a instalação do SCGR-IAG, diferentes métodos geofísicos de investigação rasa estão sendo avaliados quanto ao desempenho e a confiabilidade quando aplicados em problemas urbanos, visando o mapeamento de interferências em subsuperfície, estudos de contaminação do meio ambiente e em pesquisas arqueológicas (Borges et al., 2004; Porsani et al., 2004b; Rodrigues, 2004; Rodrigues et al., 2004; Silva et al., 2004).

\section{Recuperação da paisagem}

Após a instalação dos alvos, uma equipe de jardinagem cuidou de reparar as irregularidades do terreno e do plantio de gramas, recuperando a paisagem local. Esta atividade é importante para que nenhuma evidência dos alvos instalados fique visível na su- 
perfície. As únicas evidências na superfície são os quatro marcos de concreto instalados nos vértices do SCGR. A Figura 7 mostra uma foto da área depois de 14 meses da instalação do Sítio Controlado.

\section{ATIVIDADES DESENVOLVIDAS "DEPOIS" DA INSTALA- ÇÃO DO SCGR-IAG \\ Aquisição dos dados geofísicos e processamento dos dados GPR}

Até 0 presente momento foram adquiridos perfis GPR, de caminhamento elétrico e de condutividade elétrica aparente com 0 método Eletromagnético Indutivo (EM38). Esta é uma atividade dinâmica e contínua, pois a área do SCGR será utilizada no desenvolvimento das práticas de campo dos alunos de graduação e de pós-graduação em Geofísica do IAG. Vale ressaltar que os trabalhos práticos a serem realizados na área do SCGR não excluirão as fundamentais atividades de campo.

Neste trabalho são mostrados os resultados de 51 perfis GPR, adquiridos com as antenas blindadas de $250 \mathrm{MHz}$ e polarização do campo elétrico-Ey. Normalmente, a componente Ey das antenas são posicionadas perpendiculares à direção do perfil, visando obter o máximo acoplamento entre os sinais transmitidos e recebidos (Versteeg, 1996). Os perfis foram adquiridos no modo contínuo, com stack de 32, na direção N-S, o intervalo entre os traços foi de $5 \mathrm{~cm}$ e espaçamento entre os perfis de $1 \mathrm{~m}$.

0 processamento dos dados GPR foi realizado utilizandose 0 software Radan (GSSI). As principais etapas utilizadas foram: correção do tempo zero, análise espectral, filtragem temporal passa banda, ganhos no tempo (linear, constante, esférico e exponencial), migração-fk e conversão tempo/profundidade. Para a migração e a conversão de tempo para profundidade foi utilizada a velocidade de 0,065 m/ns, que corresponde aos soIos argilo-arenosos da área do SCGR. Esta velocidade foi calculada com base na medida do tempo-t (lido no radargrama) e na profundidade-h ao topo de um dos tambores metálicos. De posse dos valores de $t \mathrm{e} h$, basta substituí-los na expressão de tempo de trânsito $(v=2 h / t)$ para se obter a velocidade de propagação da onda eletromagnética no meio.

\section{Modelagem numérica GPR 2D}

Para dar mais confiabilidade à interpretação e análise dos perfis GPR sobre os tambores metálicos, estudos complementares de modelagem numérica GPR 2D foram realizados utilizando-se 0 software ReflexW (Sandmeier, 2004). A simulação da propagação da onda eletromagnética num modelo 2D foi baseada na solução das equações de Maxwell através do método das diferenças finitas no domínio do tempo (FDTD) (Ward \& Hohmann, 1987).

0 Método FDTD consiste na implementação de um algoritmo computacional através de aproximações numéricas sucessivas, substituindo as equações diferenciais por diferenças finitas. A substituição se dá pela expansão das equações diferenciais em Série de Taylor e truncamento na segunda ordem. Desta forma, as equações de Maxwell são resolvidas por equaç̧ões algébricas, através do conceito de discretização desenvolvido por Euler, na qual as taxas relacionadas às derivadas são substituídas por incrementos espaciais e temporais. 0 modelo é discretizado em um grid composto por um conjunto finito de pontos representados pelos chamados nós da malha, cada nó representa um vetor de campo com propriedades elétricas e magnéticas (Costa, 2002).

Dentre os trabalhos de simulações numéricas GPR 2D através do método FDTD sobre alvos instalados em subsuperfície temse: Chen \& Huang (1998), Hammon III et al. (2000), Lampe et al. (2003), Rodrigues (2004), Rodrigues et al. (2004), dentre outros. Zeng \& McMechan (1997) utilizaram o método do traçado de raios para simular tanques e canos enterrados, e obtiveram interessantes resultados. As modelagens numéricas são de grande importância para se preverem os resultados a serem obtidos numa situação real de campo e também servem para caracterizar os alvos em subsuperfície, através de um estudo comparativo entre os resultados real e 0 sintético.

Para as simulações dos perfis GPR foram usadas antenas dipolares de $250 \mathrm{MHz}$ e polarização do campo elétrico-Ey. 0 campo de ondas gerado foi simulado utilizando uma fonte do tipo exploding reflector, no qual as ondas são geradas simultaneamente a partir do alvo e enviadas para a superfície (Yilmaz, 1987; Daniels, 1996). Este procedimento corresponde ao reposicionamento das hipérboles de difração nos alvos, colapsando a energia para 0 ápice da hipérbole, sendo um procedimento comum na etapa de migração de dados GPR e sísmicos.

\section{RESULTADOS GPR}

A Figura 8a mostra a distribuição espacial dos tambores metálicos vazios instalados na Linha 4 do SCGR-IAG. Nesta linha foram enterrados sete tambores dispostos na horizontal e na vertical, em profundidades de 0,5, 1 e 1,5 $\mathrm{m}$ de profundidade em relação ao topo dos tambores. Além disso, um cano metálico-guia de $4 \mathrm{~cm}$ de diâmetro foi enterrado a 0,5 $\mathrm{m}$ de profundidade.

A Figura 8b mostra 0 resultado da modelagem numérica GPR 2D dos tambores metálicos. Note que somente 0 topo dos alvos é determinado pela simulação, sendo caracterizado 


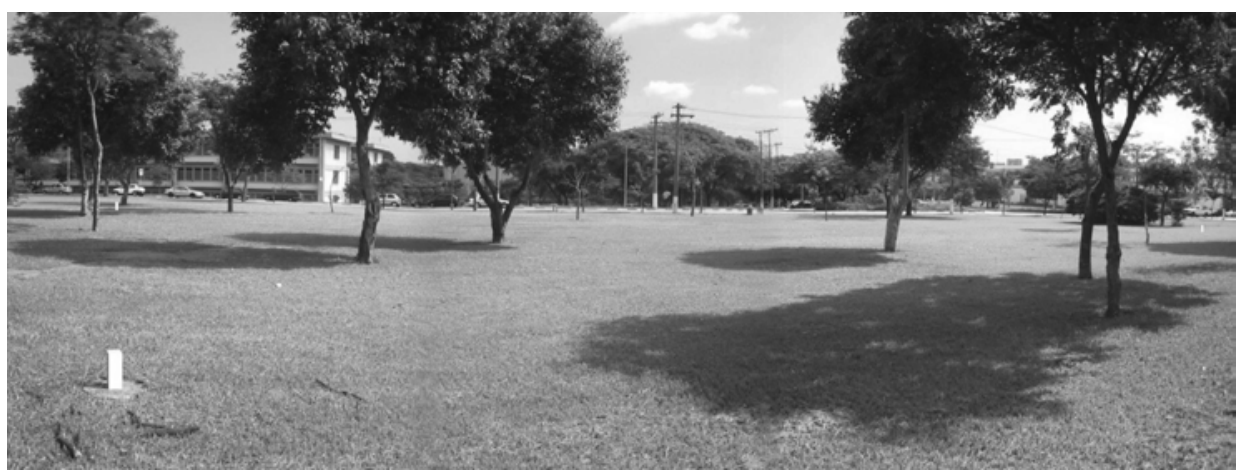

Figura 7 - Área do SCGR-IAG depois de 14 meses da instalação dos alvos

por fortes refletores hiperbólicos devido à elevada condutividade do metal $\left(10^{+9} \mathrm{mS} / \mathrm{m}\right)$. Neste caso, praticamente toda a energia da onda eletromagnética é refletida no metal. Devido à utilização da fonte do tipo exploding reflector, nota-se a presença de algumas difrações abaixo das hipérboles que são provocadas pela reverberação da onda eletromagnética nos tambores. Esta reverberação tem maior intensidade nos tambores mais rasos, devido a maior intensidade do sinal na parte superior do subsolo. Em antenas de maior freqüência esta reverberação torna-se mais intensa devido ao menor comprimento de onda. Observa-se que o padrão de reflexão nos tambores horizontais é diferente dos tambores verticais, ou seja, a forma do alvo influi no padrão de reflexão.

A Figura 8c mostra o resultado de um perfil GPR de $250 \mathrm{MHz}$ realizado sobre os tambores metálicos. Observa-se que o topo dos tambores metálicos foi caracterizado por uma forte reflexão hiperbólica, o que já era esperado conforme mostrado no resultado da modelagem numérica (Figura 8b). 0 cano metálico-guia, situado na posição $15 \mathrm{~m}$, é caracterizado pelo refletor hiperbólico mais apertado. Note que a partir de 2,5 $\mathrm{m}$ de profundidade 0 sinal GPR é fortemente atenuado devido às características condutivas dos sedimentos da Bacia de São Paulo (Porsani et al., 2004a; Borges et al., 2004).

A Figura 8d mostra o perfil GPR de $250 \mathrm{MHz}$ após a migração-fk, utilizando velocidade constante de $0,065 \mathrm{~m} / \mathrm{ns}$. Observase que as reflexões no topo de alguns tambores metálicos e no cano metálico-guia foram colapsadas num ponto, e em outros, as reflexões ficaram mais apertadas. Este fato deve-se às heterogeneidades nas propriedades físicas do solo em subsuperfície, portanto, o meio não é uniforme e a velocidade da onda varia tanto lateralmente quanto verticalmente.

A Figura 9 mostra o resultado de um bloco diagrama GPR 3D para as antenas de $250 \mathrm{MHz}$ sobre toda a área do SCGR. Esta fi- gura foi obtida a partir da interpolação dos perfis GPR 2D, sendo uma maneira de visualizar em $3 \mathrm{D}$ o conjunto de dados dentro do SCGR. A partir de um cubo sólido 3D também pode-se apresentar os resultados na forma de diagrama de cercas para as linhas $4 \mathrm{e}$ 7 instaladas no SCGR (Figura 10). Uma outra forma interessante de apresentar os resultados é na forma de depth slices. A Figura 11 mostra um depth slice em 0,5 m de profundidade. Observase claramente a presença de uma anomalia linear na posição de $15 \mathrm{~m}(\mathrm{~N}-\mathrm{S})$ e com $50 \mathrm{~m}$ de comprimento (E-W), correspondente ao cano metálico-guia; e 0 alvo denominado de $A$, correspondente ao tambor metálico disposto a $0,5 \mathrm{~m}$ de profundidade conforme mostrado na Figura 8a. As outras anomalias que ocorrem ao longo da posição de $38 \mathrm{~m}$ (E-W) correspondem aos tambores de plásticos (Linha 5 do SCGR) e que serão analisadas posteriormente.

\section{CONCLUSÕES}

A descrição das principais etapas envolvidas na instalação do Sítio Controlado de Geofísica Rasa-SCGR do IAG/USP poderá servir de referência para a construção de novos campos de prova no Brasil, assim como outros pré-existentes guiaram a instalação deste sítio.

A caracterização geofísica, sob condições controladas no SCGR-IAG, permitiu uma calibração das respostas geofísicas para tambores metálicos. Este tipo de caracterização deverá servir para testar os diversos métodos geofísicos que são rotineiramente utilizados em estudos geológicos, geotécnicos, ambientais e arqueológicos.

As assinaturas geofísicas dos tambores metálicos poderão ser usadas como respostas padrões típicas e serem extrapoladas para áreas onde não se têm informações da subsuperfície.

Os estudos de modelagem numérica GPR 2D pelo método das diferenças finitas no domínio do tempo se mostraram muito 


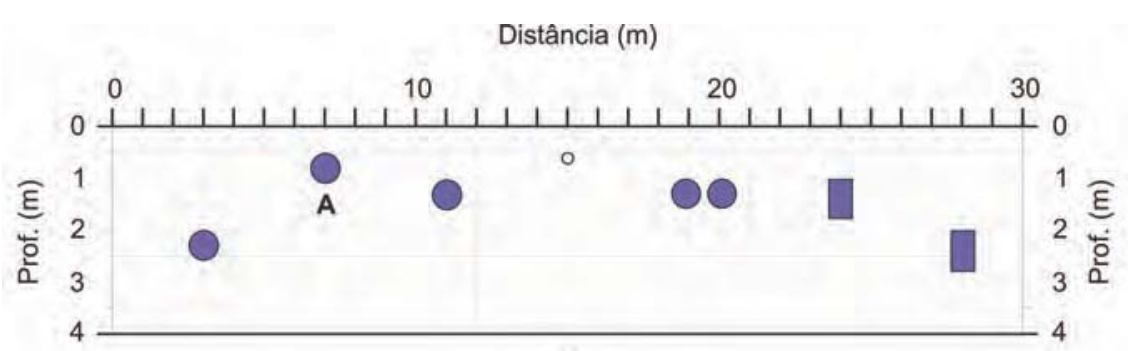

(a)

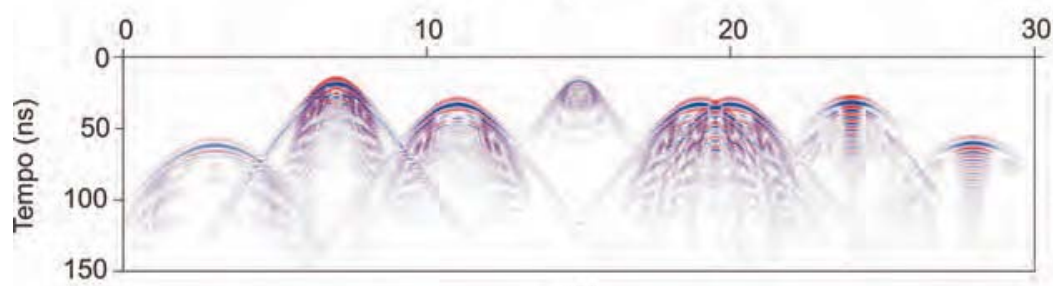

(b)

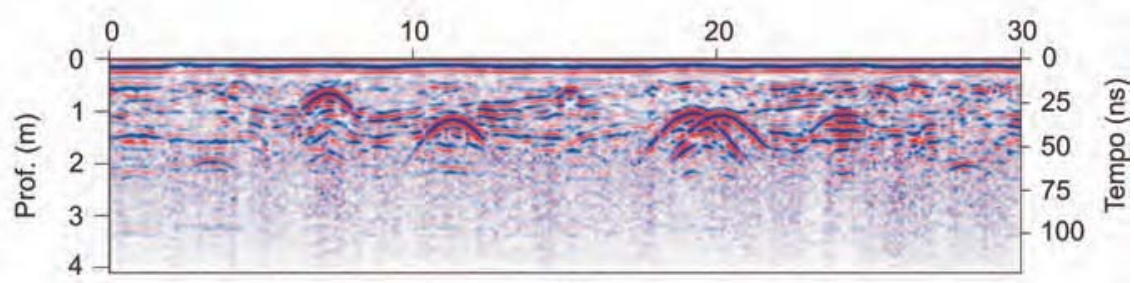

(c)

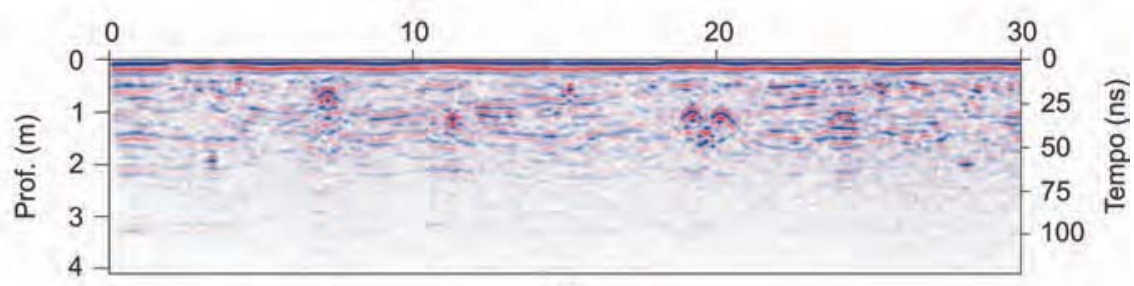

(d)

Figura 8 - a) Distribuição espacial dos tambores metálicos vazios instalados na Linha 4 do SCGR-IAG; b) Perfil GPR sintético de $250 \mathrm{MHz}$ resultante da modelagem numérica 2D; c) Perfil GPR de $250 \mathrm{MHz}$ antes da migração; d) Perfil GPR de $250 \mathrm{MHz}$ migrado com velocidade constante de $0,065 \mathrm{~m} / \mathrm{ns}$.

importantes para se prever os resultados reais e comprovar as anomalias e os padrões típicos de reflexões sobre os alvos cujas propriedades físicas são conhecidas.

0 perfil de $250 \mathrm{MHz}$ apresentou uma excelente concordância com o modelo GPR 2D, e a migração melhorou a resolução e 0 posicionamento espacial dos tambores metálicos. Os resultados GPR na forma de cubo 3D, diagrama de cercas e depth slices foram importantes para melhorar a caracterização dos alvos em subsuperfície.

Para finalizar, o Sítio Controlado do IAG se constitui numa importante ferramenta para o Ensino, Pesquisa e a Extensão Universitária em Geofísica, e a sua instalação permitiu melhorar 0 conhecimento relativo às respostas geofísicas de alvos rasos.

\section{AGRADECIMENTOS}

Ao IAG-USP pelo apoio e pela infra-estrutura. Ao IF/USP pela autorização para instalar o SCGR-IAG. À FAPESP (Processos 02/07509-1 e 02/07510-0) pelo fundamental apoio financeiro para a instalação do SCGR e pela bolsa de doutorado de Welitom Rodrigues Borges. À CAPES pela bolsa de mestrado de Selma Isabel Rodrigues. A prefeitura do campus da USP pelo empréstimo da retroescavadeira. À SABESP pela doação dos tubos metálicos e de PVC. À ELETROPAULO pela doação dos cabos elétricos e conduítes de plástico. Aos colegas do IAG Carlos AI- 


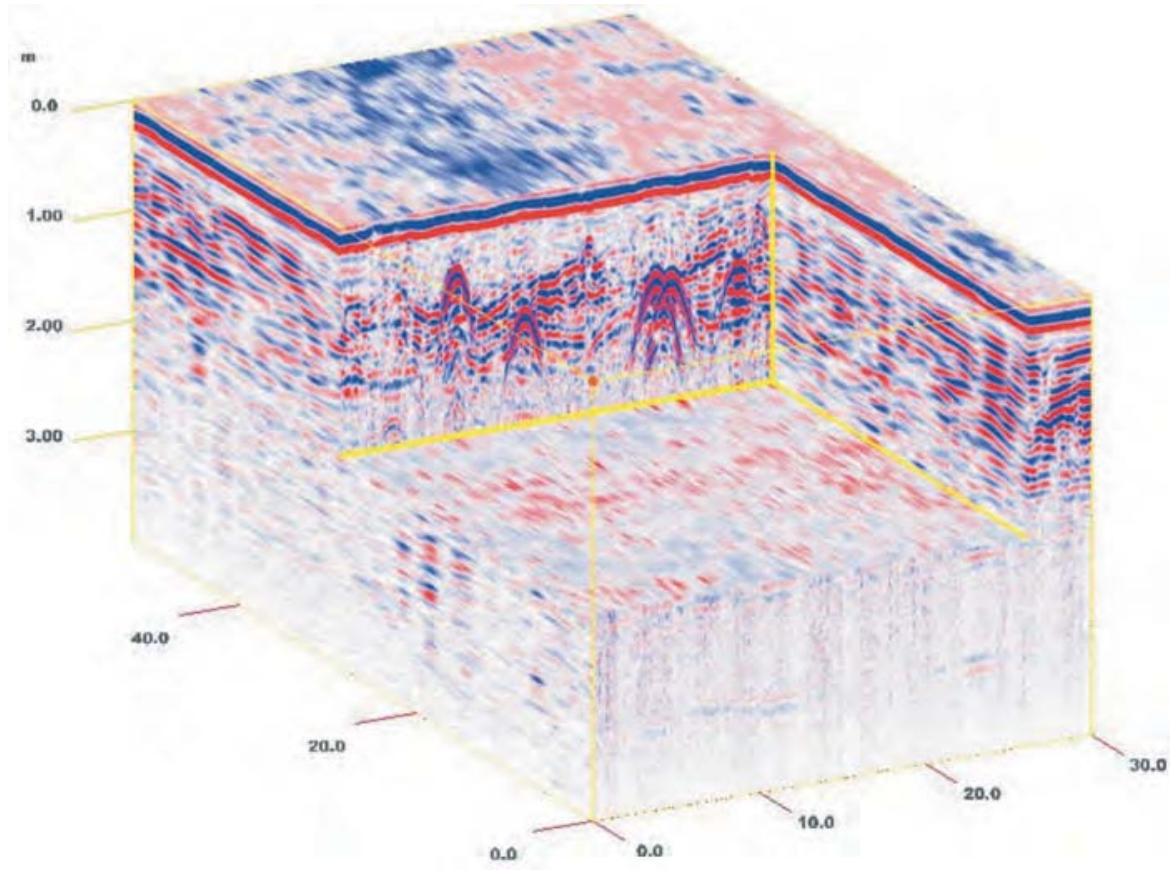

Figura 9 - Cubo GPR 3D para as antenas de $250 \mathrm{MHz}$ sobre toda a área do SCGR-IAG.

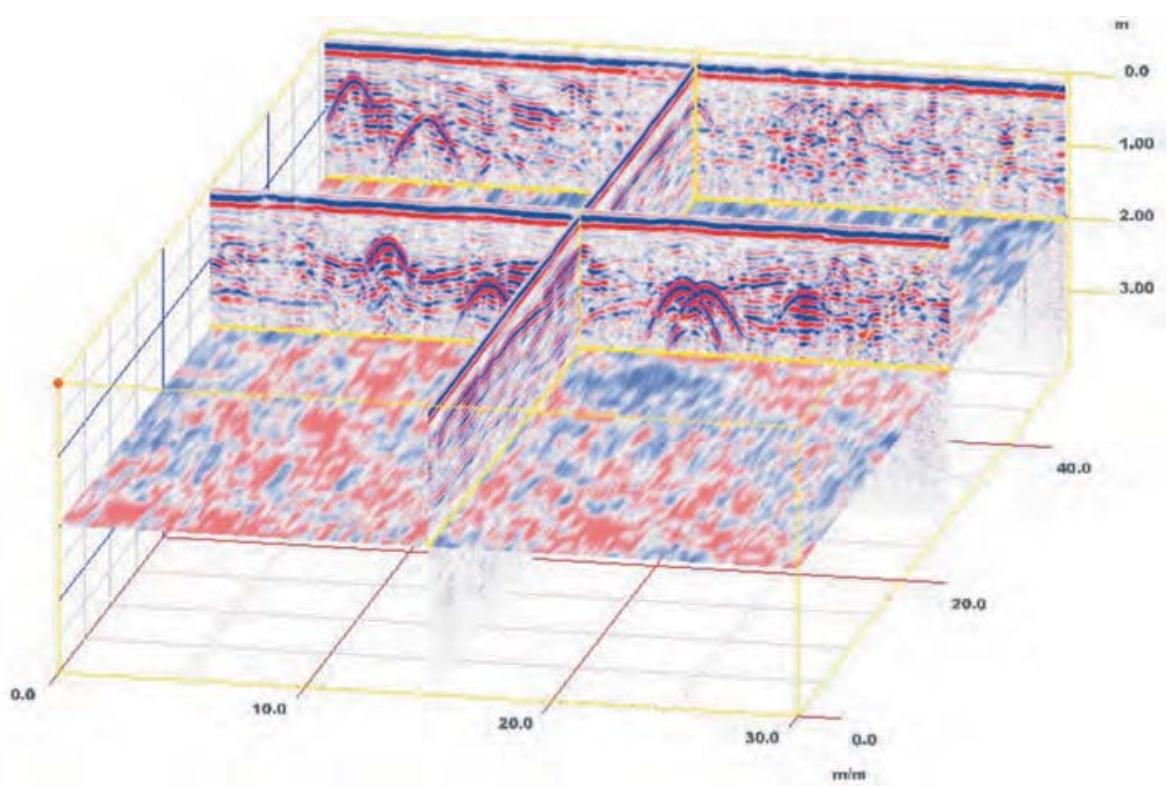

Figura 10 - Diagrama de cercas dos perfis GPR de $250 \mathrm{MHz}$ para as linhas 4 e 7 instaladas no SCGR-IAG.

berto Mendonça, Vagner Roberto Elis, Renato Luiz Prado, Liliana Alcazar Diogo e Marcelo Sousa de Assumpção pelas proveitosas discussões e sugestões, e ao "anônimo" assessor da FAPESP pelas valiosas sugestões, que muito contribuíram para o sucesso da instalação do SCGR-IAG/USP. Ao IPT pelo empréstimo da antena de $250 \mathrm{MHz}$. Ao Ernande Costa Santos e aos alunos da Graduação e da Pós-Graduação em Geofísica do IAG pela fundamental ajuda na instalação dos alvos e na aquisição dos dados geofísicos. Finalmente agradecemos aos revisores da RBGf pelas proveitosas sugestões. 


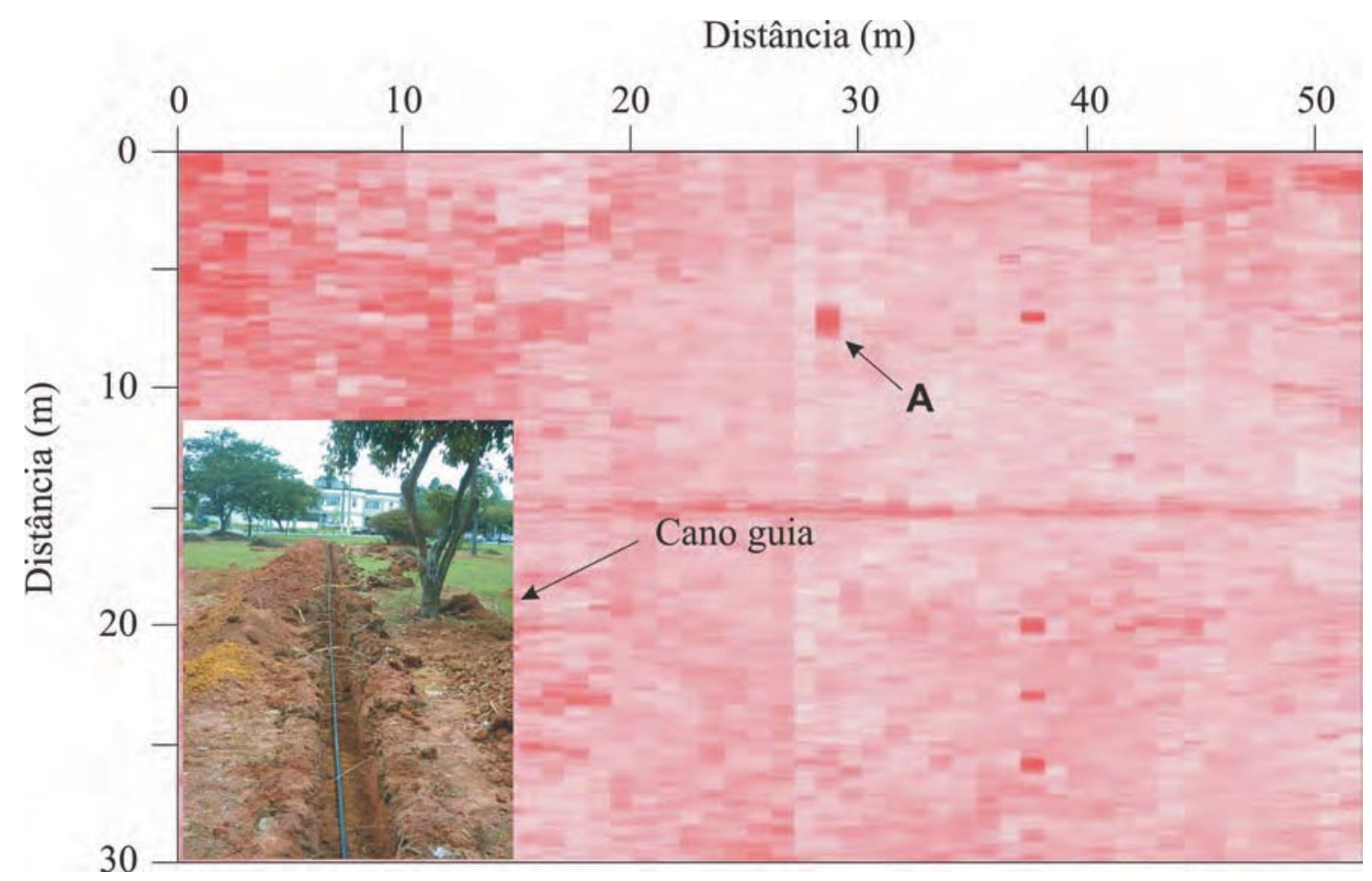

Figura 11 - Depth slice em 0,5 m de profundidade para as antenas de $250 \mathrm{MHz}$.

\section{REFERÊNCIAS}

BORGES WR, PORSANI JL, SILVA EMA, ELIS VR \& HIODO FY. 2004. Utilização de Tomografia Elétrica na Identificação da Interface entre uma Cobertura Antrópica e Sedimentos Fluviais na Área do Sítio Controlado de Geofísica Rasa do IAG/USP. In: Simpósio Brasileiro de Geofíisica, I, São Paulo: SBGf. CD-ROM.

CHEN HW \& HUANG TM. 1998. Finite Difference Time Domain Simulation of GPR Data. Journal of Applied Geophysics, 40: 139-163.

COSTA FP. 2002. Solução numérica de equações diferenciais. Mini curso. Departamento de Ciências Exatas e Tecnológicas, Universidade Estadual de Santa Cruz, Santa Catarina, 22 p.

DANIELS DJ. 1996. Surface Penetrating Radar. The Institution of Eletrical Engineers, London, United Kingdom. 300 p.

DAVIS JL \& ANNAN AP. 1989. Ground penetrating radar for high resolution mapping of soil and rock stratigraphy. Geophysical Prospecting, 37: $531-551$.

DIOGO LA, LE DIAGON FMM \& PRADO RL. 2004. Bedrock imaging using post-critical shallow seismic reflection data. Journal of Applied Geophysics, 57: 1-9.

HAMMON III WS, MCMECHAN GA \& ZENG X. 2000. Forensic GPR: Finite-Difference Simulations of Responses from Buried Human Remains. Journal of Applied Geophysics, 45: 171-186.
LAMPE B, HOLLIGER K \& GREEN AG. 2003. A Finite Difference Time Domain Simulation Tool for Ground Penetrating Radar Antennas. Geophysics, 68(3): 971-987.

PORSANI JL. 1999. Ground Penetrating Radar (GPR): Proposta Metodológica de Emprego em Estudos Geológico-Geotécnicos nas Regiões de Rio Claro e Descalvado - SP. Tese (Doutorado), Instituto de Geociências e Ciências Exatas, Universidade Estadual Paulista, Rio Claro - SP, 145 p.

PORSANI IL, BORGES WR, ELIS VR, DIOGO LA, HIODO FY, MARRANO A \& BIRELLI CA. 2004a. Investigações Geofísicas de Superfície e de Poço no Sítio Controlado de Geofísica Rasa do IAG-USP. Revista Brasileira de Geofísica, 22(3): 245-258.

PORSANI JL, RODRIGUES SI, BORGES WR \& HIODO FY. 2004b. 0 Sítio Controlado de Geofísica Rasa do IAG-USP: Instalação e Resultados Preliminares. In: Simpósio Brasileiro de Geofísica, I, São Paulo: SBGf. CDROM.

RODRIGUES SI. 2004. Caracterização GPR de Tambores Metálicos e Plásticos Rasos: Estudo sobre o Sítio Controlado do IAG-USP. Dissertação (Mestrado em Geofísica), Instituto de Astronomia, Geofísica e Ciências Atmosféricas, Universidade de São Paulo, São Paulo, 89 p.

RODRIGUES SI, PORSANI JL, BORGES WR \& HIODO FY. 2004. Emprego do GPR no Mapeamento de Tambores Plásticos Enterrados no SCGR do IAG. In: Simpósio Brasileiro de Geofísica, I, São Paulo: SBGf. CD-ROM. 
SANDMEIER KJ. 2004. ReflexW Version 3.5. Program for the Processing of Seismic, Acoustic or Electromagnetic Reflection, Refraction and Transmission Data.

SAUCK W. 1996. Controlled Site for Shallow Geophysics. Institute for Water Sciences, Department of Geosciences, Western Michigan University. Disponível em: <http://www.geology.wmich.edu/fhydro/>. Acesso em: 10 maio 2005.

SILVA EMA, ELIS VR, BORGES WR, PORSANI JL \& HIODO FY. 2004. Utilização de Tomografia Elétrica na Identificação de Tambores Metálicos Implantados na Área do Sítio Controlado de Geofísica Rasa do IAG/USP. In: Simpósio Brasileiro de Geofísica, I, São Paulo: SBGf. CD-ROM.
VERSTEEG R. 1996. Optimization of GPR Acquisition and Noise Elimination Parameters. In: $6^{\text {th }}$ International Conference on Ground Penetrating Radar, (GPR'96), Sendai, Japan, p. 289-292.

WARD SH \& HOHMANN GW. 1987. Electromagnetic Theory for Geophysical Applications: Electromagnetic Methods in Applied Geophysics. Tulsa: Society of Exploration Geophysicists, NABIGHIAN MN (Ed.). 1(3): 131-311.

YILMAZ 0. 1987. Seismic Data Processing. Tulsa: Society of Exploration Geophysicists Press. $526 \mathrm{p}$

ZENG X \& McMECHAN GA. 1997. GPR Characterization of Buried Tanks and Pipes. Geophysics, 62(3): 797-806.

\section{NOTAS SOBRE OS AUTORES}

Jorge Luís Porsani. Geólogo pelo Instituto de Geociências da UFBa (1987). Mestrado em Geofísica pelo Núcleo de Pesquisas Geofísicas Aplicadas à Prospecção de Hidrocarbonetos da UFPa (1991). De 1991 a 1996, trabalhou como Geofísico no Centro de Pesquisas da PETROBRAS. Doutorado em Geociências e Meio Ambiente pelo Instituto de Geociências e Ciências Exatas da UNESP (1999). Pós-Doutorado na Western Michigan University-USA no período de julho-novembro/2005 (Fapesp 04/13609-4). Desde 01/12/1998 é Docente do Departamento de Geofísica do IAG/USP, atuando com métodos elétricos e eletromagnéticos (GPR) aplicados à geologia, geotecnia, exploração mineral, meio ambiente e arqueologia. Coordenou o Projeto de Instalação do Sítio Controlado de Geofísica Rasa-SCGR do IAG/USP (Fapesp 02/07509-1).

Welitom Rodrigues Borges. Geólogo pelo Instituto de Ciências Exatas e da Terra da Universidade Federal de Mato Grosso (ICET/UFMT) em 2000. Em 2002 obteve 0 título de Mestrado em Ciências, área de Geofísica Aplicada, pelo Instituto de Astronomia, Geofísica e Ciências Atmosféricas da Universidade de São Paulo (IAG/USP). Atualmente está fazendo o Doutorado (bolsista Fapesp 02/07510-0) junto ao Programa de Pós-Graduação em Geofísica do IAG/USP, desenvolvendo a pesquisa "Caracterização geofísica de alvos rasos com aplicações no planejamento urbano, meio ambiente e arqueologia: Estudo sobre o sítio controlado do IAG/USP".

Selma Isabel Rodrigues. Formada em Tecnologia Mecânica pela FATEC-SP, 2001. Mestra em Ciências, área de Geofísica Aplicada, pelo Instituto de Astronomia, Geofísica e Ciências Atmosféricas da Universidade de São Paulo - IAG/USP (2004). Em 2004, trabalhou como Geofísica junto a Empresa IDS-Radar Ltda utilizando métodos de investigação rasa, elétricos e eletromagnéticos (GPR). Atualmente, é aluna de doutoramento do Programa de Pós-Graduação em Geofísica do IAG/USP, desenvolvendo a pesquisa "Caracterização geofísica de sítios arqueológicos de sambaquis fluviais na Região de Santa Catarina, utilizando os métodos GPR e eletromagnético indutivo" (bolsista Fapesp 04/13191-0).

Francisco Yukio Hiodo. Graduado em Física pelo Instituto de Física da Universidade de São Paulo-USP em 1972. Mestrado em Geofísica pelo Instituto de Astronomia, Geofísica e Ciências Atmosféricas (IAG-USP) em 1981. Doutorado em Geofísica pelo IAG-USP em 1990. Auxiliar de Ensino no IAG-USP em 1973. Professor Assistente em 1981. Professor Doutor do IAG-USP desde 1990. Atualmente é especialista em Instrumentação Geofísica nas áreas de Paleomagnetismo (magnetômetros rotativos e desmagnetizadores), Geofísica Nuclear (espectrômetros gama e emanômetros de radônio), Perfilagem de poços tubulares, Geotermometria e sensores de temperatura, Magnetômetros de precessão de prótons e fluxgate, Métodos geoelétricos e eletromagnéticos. 